\title{
Gubernamentalidad y relaciones de inclusión/ exclusión: los dispositivos de intervención social dirigidos a mujeres inmigradas en España*
}

\author{
Governmentality and Relations of Inclusion/Exclusion: Social \\ Intervention Devices Aimed at Immigrant Women in Spain
}

Recibido: 3 de noviembre de 2014 | Aceptado: 17 de agosto de 2015

\author{
Caterine Joanna Galaz Valderrama** \\ Universidad de Chile, Santiago, Chile \\ MARISEla MONTENEGRO MARTÍNEZ *** \\ Universidad Autónoma de Barcelona, Cataluña, España
}

doi : 10.11144/Javeriana.up14-5.grie

Para citar este artículo: Galaz, J. C. y Montenegro, M. (2015). Gubernamentalidad y relaciones de inclusión/exclusión: los dispositivos de intervención social dirigidos a mujeres inmigradas en España. Universitas Psychologica, 14(5), 1667-1680.

http://dx.doi.org/Javeriana.upsy14-5.grie

* Artículo de investigación realizado con base en los resultados obtenidos de los proyectos "Mujeres inmigrantes y sistema educativo en la sociedad receptora: análisis y propuestas del mejoramiento del acceso y la satisfacción en los recursos de formación para la inserción sociolaboral" (Instituto de la mujer. Ministerio de trabajo y asuntos sociales 2007-2009); "Polítiques, pràctiques i estratègies d'inserció: Una mirada des de les trajectòries de les dones immigrades" (ARAFI 2010-2011); y "Significats de la inserció sociolaboral i processos d'intervenció social amb dones migrades en el territori català" (Institut Català de les Dones 2010-2011). Por otro lado, reflexiones extraídas de la investigación, financiada por la Vicerectoría de Investigación (VID), de la Universidad de Chile, U. Inicia: "Intervención social con mujeres inmigradas: Reconocimiento, incorporación sociocultural e igualdad de oportunidades para la inclusión" (2014-2016).

** Doctora en Ciencias de la Educación, UAB. Miembro del Programa Psicología Social de la Memoria y académica de Trabajo social, Universidad de Chile. Máster en Políticas Sociales y Gestión Local (Arcis).Correo electrónico: cgalazvalderrama@u. uchile.cl

${ }^{* * * *}$ Licenciada en Psicología, Universidad Central de Venezuela. Doctora en Psicología Social, Universidad Autónoma de Barcelona, España. Profesora titular interina, Departamento de Psicología Social. Correo electrónico: marisela.montenegro@uab.cat

\section{RESUMEN}

Las migraciones internacionales se han erigido como un campo de preocupación de los procesos de control y gobierno en las sociedades europeas contemporáneas, conformándose como "problema social" que es preciso atender desde diferentes estamentos públicos. De la necesidad de gestión de estos grupos poblacionales emergen leyes, reglamentos, discursos, planes de actuación y procesos de intervención cuyo efecto principal es la reproducción y solidificación de las relaciones de poder presentes en las sociedades de instalación. A partir de diversas investigaciones en el campo de los servicios que se prestan a mujeres inmigradas en el Estado español, se analizan las maneras en las que se instalan mecanismos de gubernamentalidad que contribuyen a la construcción de una "otra problemática y carente" que es necesario atender para su correcta integración social; mecanismos que ayudan a legitimar la necesidad de adaptación de estas personas a un contexto de desigualdad jurídica, social y cultural que resulta incuestionado. Palabras clave

gubernamentalidad; intervención social; migraciones trasnacionales; interseccionalidad

\section{A B S T R A C T}

International migration has emerged, in contemporary European societies, as an area of concern for control and governmentality processes, by means of its definition as a "social problem" that must be addressed by public organisms. Different laws, regulations, discourses, plans and intervention processes emerge from the need to manage these populations, generating as main effect the reproduction and solidification of power relations in host societies. Departing from different researches developed in the field of services provided to immigrant women in Spain, we propose to analise the ways in which governmentality mechanisms contribute to construct a subject that is understood as an "Other problematic and needing" that requires attention to achieve proper social integration. These mechanisms contribute to justify the urge for this population to adapt to a context of legal, social and cultural inequality that results unquestioned.

Keywords

governmentality; social intervention; transnational migrations; intersectionality. 


\section{La intervención social como mecanismo de gubernamentalidad}

El principio que sostiene el campo de la intervención social es la necesidad de transformar situaciones que se entienden como problemáticas. Sin embargo, la definición de qué es intervenible, y las formas de abordarlo, es un asunto controvertido que se enmarca en definiciones y redefiniciones en las cuales están involucradas tanto instituciones y agentes sociales como conocimientos y formas de acción que se ponen en práctica en contextos concretos (Montenegro \& Balasch, 2011). Estos procesos de definición se dan en una trama de relaciones de poder conformada por discursos, leyes, corrientes de pensamiento y metodologías de intervención en la que emergen ciertas construcciones mientras otras quedan latentes o desaparecen (Carballeda, 2010); regímenes de inteligibilidad donde se vinculan las prácticas de gobierno y dominio de las poblaciones con el conocimiento científico, y que resultan convenientes para determinados órdenes sociales (Álvarez, 2008; Sandoval, 1997). Este conjunto de prácticas y operaciones de gobierno permiten constituir, definir y organizar las poblaciones, de tal modo que no sea necesario recurrir al ejercicio de la fuerza y la coerción -propio del paradigma jurídico- para ejercer poder (Foucault, 1975 a). En ese sentido partimos de la noción de gubernamentalidad entendida como:

"el conjunto constituido por las instituciones, los procedimientos y las tácticas que permiten ejercer esta forma tan específica, tan compleja, de poder, que tienen como meta principal la población, como forma primordial el saber, como instrumento técnico esencial, los dispositivos de seguridad" (Foucault, 1999, p. 195).

Los mecanismos de gubernamentalidad, entonces, buscan moldear la conducta de los individuos y colectivos, no solamente a través del control, la disciplina o la normalización, sino también por medios más sutiles y no directamente coercitivos que los hacen más inteligentes, contentos, virtuosos, saludables, productivos, dóciles, emprendedores, etc. (Rose, 1996). La intervención social entra de lleno en la lógica de la gubernamentalidad, en el sentido de que genera los mecanismos por los cuales se producen conocimientos, técnicas y personas expertas que tienen como objetivo adentrarse en las vidas de las poblaciones a partir justamente de una posición institucional legitimada socialmente. Es a través del ejercicio de poder, con regulaciones sutiles que se producen en el espacio de la intervención social, como se desarrollan dinámicas relacionales entre sujetos interventores e intervenidos. Así, considerando la noción de gobierno de Foucault, se refiere a la conducción de las conductas (Grinberg, 2007), que implica el cómo se ejerce la autoridad de unas sobre otras y sobre sí mismas. La intervención social constituye uno de los nodos de la matriz desde el que emergen las aproximaciones en torno a fenómenos, colectivos o territorios señalados como afectados por los problemas sociales y sobre los que se busca promover algún cambio social como, por ejemplo, la inmigración y en particular, las mujeres migrantes.

Por ello, en este artículo se discuten algunas maneras como se constituye el fenómeno de la migración trasnacional femenina como problema social en el contexto europeo y, en este sentido, como objeto de gobierno sobre el cual se diseñan e implementan proyectos de intervención social. De esta manera, a partir de tres investigaciones llevadas a cabo en el Estado español, que estudiaron los servicios dirigidos a mujeres inmigrantes como dispositivos, se analizarán las operaciones concretas a través de las cuales se materializan discursos y prácticas de gobierno que intersectan las diferenciaciones de género, etnia/origen nacional, condición socioeconómica, entre otras, lo que reproduce las relaciones de dominación presentes en las sociedades de recepción.

\section{Construcción de la inmigración internacional como "problema social"}

En el caso de los sujetos migrantes, las prácticas de gubernamentalidad dadas a través de la intervención social están supeditadas a un a priori: la consideración de la "migración" como un proble- 
ma (Blanch, 2004; Callén \& Montenegro, 2004). Las migraciones trasnacionales se erigen como un "problema de Estado" en el contexto europeo desde hace ya algunas décadas (Yufra, 2013), emergiendo una "otredad extraña" a lo que se considera como "normal" (Goffman, 2006). Para construir la figura del "migrante como problema" en las sociedades de recepción ha sido necesario un mecanismo de ejercicio de poder doble: de diferenciación, por un lado, y de subalternización, por el otro. Una operación de diferenciación que se reproduce de manera sistemática a través de prácticas institucionales y de discursos sociales, culturales y políticos (Brah, 1992), en los cuales la figura del migrante se entiende como diferente respecto de una supuesta comunidad de iguales, conformada por personas autóctonas, en la cual se quiere instalar, y en relación de asimetría respecto de los miembros del grupo mayoritario, legítimos moradores de la sociedad de instalación (Romero Bachiller, 2006; Santamaría, 2002). Y una operación de subalternización que vendría a indicar la dinámica histórica, social y cultural entre los grupos hegemónicos y las personas que son sometidas a esta mayoría y la posibilidad efectiva que poseen de autonomía y resistencia. Siguiendo a Dube (2001), este proceso de subalternización conlleva a que los sujetos sean apartados, de forma intencional o no, hacia los márgenes del sistema social desde una posición hegemónica. El sujeto que es subalternizado sería quien está subordinado, puesto en situación de inferioridad o bien instrumentalizado por otras personas, y aludiría a una situación de dominio, de clase, política, relacional y cultural. De este modo, la categorización de "inmigrante" es el producto de un proceso social, político, económico y de transacciones simbólicas que le ubica en los márgenes del sistema, en una calificación de diferencia y en una posición subalterna.

Es a través de este doble proceso de diferenciación y subalternización como la figura del migrante es entendida como un "problema social" tanto en términos de amenaza, competencia y causante de conflictividad social como en términos de una persona con múltiples problemáticas económicas, sociales y de incompatibilidad "cultural" (Blanch, 2004; Santamaría, 2002). En consecuencia, se ha- cen presentes diversos sistemas de gobierno en los procesos de instalación de las personas inmigrantes, siendo gestionados a través de diferentes marcos públicos de actuación: desde las políticas de control de seguridad que legitiman la necesidad de limitar los flujos migratorios y los derechos de estas personas (Sassen, 2013; Wagman, 2006) hasta las políticas sociales y procesos de intervención profesionalizada que buscan paliar las diferentes problemáticas asociadas al fenómeno a nivel comunitario (Agrela, 2004; Batsleer, Chantler, \& Burman, 2003; Gregorio Gil, 2011). Así, la generación de servicios específicos para personas extranjeras aparece "justificada políticamente", dada esta visión problematizada que las constituye simbólicamente como "minorías" y como "sujetos en falta" que deben ser apoyados en sus procesos de inclusión social para alcanzar el estándar de la mayoría (Montenegro, Galaz, Yufra, \& Montenegro, 2011). Para esto, se desarrollan diversas acciones discursivas y pragmáticas de control, disciplina y normalización.

Por otra parte, cabe señalar que parte de la reflexión teórica inicial en Europa sobre el fenómeno migratorio se ha caracterizado por un sesgo masculinista, reproduciendo los imaginarios de género de las sociedades receptoras (Juliano, 1994; Nash, 2005). Como señala Gregorio Gil (2012), existe un interés creciente político y académico por establecer diversas categorías de análisis que desmantelan la idea de que el fenómeno migratorio sea un asunto solo de hombres trabajadores y restituyan la agencia de las mujeres ${ }^{1}$. En este sentido, diferentes autoras han prestado atención a las particularidades de la posición de las mujeres migrantes en estos contextos (p. ej., Gil, 2011; Gregorio Gil, 1997, 2010; Parella, 2004). Estas aproximaciones han apostado por desarrollar una perspectiva feminista, poniendo énfasis en temáticas tales como la diversidad de discriminaciones que produce la noción de "mujer inmigrante no comunitaria", el cambio en las relaciones de género y aspectos que caracterizan el movimiento trasnacional de las mujeres migrantes

1 Para profundizar en los usos políticos discursivos y los imaginarios entre mujeres e inmigración, consultar Virginia Maquieira, Carmen Gregorio Gil y Elena Gutiérrez Lima (2000). 
como también los cambios en la maternidad transnacional y la organización de los cuidados.

Con este artículo, se busca contribuir a este campo de estudio poniendo el foco en las maneras en las que se sitúa a la mujer migrante como sujeto-objeto de intervención a través de múltiples categorizaciones discursivas:

Estas categorizaciones contribuyen a producir aquello que aparentemente describen, mediante una "praxis habitualizada" (Dietz, 2003) que da lugar a unos modelos de intervención que (re)producen de forma sistemática una intervención específica con "el colectivo de mujeres inmigrantes" y "su(s) problemática(s)". (Agrela, 2004, p. 35)

Así, en las investigaciones analizadas, en lugar de diseccionar este sujeto construido como "problemático", se busca indagar en las dinámicas de los servicios dirigidos a este colectivo con el fin de verter una mirada crítica sobre las maneras en las que, desde la intervención social, se reproducen relaciones de poder respecto de las mujeres migrantes en las sociedades de recepción (Montenegro et al., 2011).

\section{Acercamiento a los servicios dirigidos a mujeres inmigrantes}

El análisis propuesto surge de las tres investigaciones citadas anteriormente, llevadas a cabo entre 2007 y 2011 en diferentes ciudades del Estado español en colaboración con 35 servicios públicos y de la sociedad civil, con recursos dirigidos a mujeres inmigrantes. Estas investigaciones tenían como objetivo común conocer y analizar los marcos de comprensión y las prácticas de intervención mediante las cuales se trabaja con mujeres inmigradas en los servicios sociales de atención (públicos y privados). Dicha línea de investigación se adscribió a un enfoque crítico de las ciencias sociales, influenciado por aportaciones socioconstruccionistas (Hacking, 1999; Ibáñez, 1994, 1996) y de las epistemologías feministas (Haraway, 1991). Estas perspectivas asumen la construcción de conocimiento como una práctica social que, como cualquier otra, está influenciada por las relaciones de dominación de la sociedad en la que se desarrolla. Se utilizaron metodologías de investigación cualitativas y un enfoque de interpretación hermenéutico. Concretamente, los procedimientos desarrollados fueron la entrevista semiestructurada y la observación participante: se llevaron a cabo observaciones en los servicios específicos dirigidos a mujeres inmigradas y se desarrollaron entrevistas semiestructuradas a profesionales y a mujeres inmigrantes usuarias (50 entrevistas semiestructuradas en total). La selección de los casos que se iban a analizar se desarrolló a partir de un muestreo teóricamente conducido (Valles, 1999), es decir que se seleccionaron a las colaboradoras a través de criterios previos establecidos por el equipo de investigación, que buscaron evidenciar diversidad y variabilidad en los tipos de servicios y experiencias migratorias de las mujeres migrantes e interventoras. En este artículo, se presentarán, a manera de ejemplo, algunos extractos de estas entrevistas y observaciones que ayudan a ilustrar la problematización planteada.

Ambos métodos permitieron tanto el acercamiento a los discursos que giran en torno a las prácticas de intervención con mujeres inmigradas y los imaginarios sobre las personas involucradas en la acción (usuarias y profesionales) como el acercamiento a las prácticas concretas de intervención (formación, información, inserción sociolaboral, asesoría, etc.). Las entrevistas semiestructuradas se realizaron a partir de guiones previos a profesionales de los servicios y a mujeres usuarias y las observaciones se realizaron en los espacios cotidianos de los servicios estudiados. Toda la documentación producida mediante estos métodos fue analizada a partir de los objetivos de investigación, tomando en cuenta tanto las categorías previas utilizadas para los guiones de las entrevistas y las observaciones como los elementos emergentes que iban surgiendo en el contacto con los servicios.

\section{Normalización en contexto de asimetría: la "integración" como norma}

Las operaciones de intervención social para con el colectivo migrante giran en torno a la noción de 
“integración", que se ha erigido como la "norma”. Si entendemos que "la norma es lo que puede aplicarse tanto a un cuerpo al que se quiere disciplinar como a una población a la que se pretende regularizar" (Foucault, 1975b, pp. 228-229), veremos que en el caso del Estado español se ha asumido esta noción como vía para hacer frente al "problema social de la inmigración". El horizonte que se pretende conseguir es el de evitar una posible "guetización cultural" en ciertos sectores sociales y geográficos y combatir discriminaciones manifiestas (Galaz, 2009) más que una integración entendida como un camino para asegurar la igualdad formal en cuanto a derechos y deberes entre las personas autóctonas e inmigradas (Subirats \& Alegre, 2007). Este llamado a la integración emerge como un trasfondo de un aparataje de gubernamentalidad que se materializa en diversas áreas y dispositivos de intervención que se han analizado a través de la aproximación a servicios sociales disponibles en el contexto español. Siguiendo a Foucault (1977), los dispositivos son una suerte de máquinas para hacer ver y para hacer hablar (visibilidad y enunciación). Esto alude a un conjunto heterogéneo de discursos, leyes, disposiciones espaciales, enunciados científicos y proposiciones morales y filosóficas, siempre inscritas en juegos de poder, que tienen por función responder a una emergencia en un determinado momento (Agamben, 2011). La pretensión de este artículo es analizar las prácticas de intervención como dispositivos, centrándonos en los efectos de control, disciplina y normalización que se generan en los servicios dirigidos a mujeres migrantes. Dichos dispositivos se conforman a partir de discursos y prácticas derivados de las leyes (como la de extranjería), los planes y programas (de acogida e integración locales como el Plan de acogida de $\mathrm{Ca}$ taluña) y las intervenciones sociales que se derivan, entre otras actuaciones que conforman la matriz de poder donde se mueven las usuarias.

La operación de normativización de la integración en estos dispositivos se da principalmente a través de tres dimensiones. En primer lugar, a nivel lingüístico, los servicios dan recursos para el aprendizaje de los idiomas vehiculares, bajo la premisa de que es la base para una interrelación con el resto de la comunidad y la propia ubicación de las usuarias dentro del sistema (especialmente aquellas reagrupadas, y en segundo término, aquellas que buscan entrar al ámbito laboral).

La profesional nos comenta que el tema del idioma es fundamental. Las que saben el idioma lo tiene más fácil, pero las otras no. Hay de todo, señala, mujeres recién llegadas hasta mujeres que llevan mucho tiempo en España pero que de todas maneras no saben la lengua porque siempre se han movido en las redes del país de origen. O hay casos que llevan años viviendo en España pero trabajan en un negocio familiar y eso las hace vivir en un micromundo que no les ayuda. Enfatiza que para poder insertarse necesitan aprender sí o sí el idioma porque si no, siempre les parecerá un mundo ajeno. (Observación participante. Servicio público)

En esta cita, se condensa el interés por promover el idioma como un imperativo de integración, de lo contrario, las propias mujeres inmigradas se condenan a la exclusión. La posibilidad de vivir en un "micromundo" o inserta en "redes del país de origen" no es entendida como una situación adecuada, pues no responde a la "norma" de integración que requiere de un esfuerzo por parte de las mujeres inmigradas para habitar en la sociedad de recepción. En este sentido, la norma de integración hace visible la diferencia para poder reintegrarla dentro de sus límites, convirtiéndose en un proyecto de disciplinamiento y control al incidir los sujetos a quienes se les aplica en este caso, las mujeres inmigradas.

En segundo lugar, encontramos otra dimensión del dispositivo de intervención basada en los exhortos a la "integración cultural". Como señalan Gregorio Gil y Franze (1999), uno de los énfasis desde los inicios de la intervención social con población inmigrante en España ha sido la elaboración de políticas sociales donde "la cultura" aparece como un instrumento de adaptación. En ese sentido, la integración se relaciona desde esas políticas como la adaptación a las pautas de la sociedad de instalación.

En las investigaciones analizadas, en la mayoría de las intervenciones sociales resulta evidente un discurso que intenta mostrar un respeto a los hechos 
culturales más explícitos (credos religiosos, formas de reunión, vestimentas, etc.). No obstante, en las prácticas de intervención que se desarrollan respecto de la "inserción al medio" de las mujeres inmigrantes se pone especial énfasis en que sean estas las que comprendan las formas de funcionamiento local y se adapten a las lógicas de la mayoría como una forma, incluso, de obtener mejores oportunidades sociales y laborales. En el ejemplo siguiente, se condensa este llamado a la normalización:

Ella era una mujer con estudios de su país, conocía varios idiomas pero usa velo y es verdad que la gente así no la va a emplear. Le explicamos que debía adaptarse a las exigencias de quienes la emplearían, para poder conseguir ese trabajo. Hay que ser realistas y se les tienen que decir las cosas para que puedan decidir cómo actuar (...). Una de nuestras funciones es decir aquello que todo el mundo piensa, pero nadie se atreve a decir, tú lo tienes que decir y después la persona verá qué hace al respecto. (Entrevista profesional. ONG)

En ese sentido, se tiende a la asimilación cultural, ya que el discurso privilegia la idea de que la manifestación de hechos culturales puede entorpecer la incorporación al mercado laboral o social (educativo, geográfico, etc.) o generar discriminaciones del resto de la sociedad por la falta de comprensión respecto de ciertos signos culturales (Herrera, 1994). Así, vemos que la responsabilidad es atribuida a la usuaria en términos de una decisión sobre cómo actuar: deberá participar activa -y libremente- en el control de su vestimenta y formas de hacer, siguiendo las recomendaciones de las profesionales de los servicios. Siguiendo a Agrela (2004), esta forma de intervención respondería a un modelo en el que la cuestión cultural se utiliza como única dimensión explicativa de las necesidades y demandas de las mujeres, en detrimento de otros procesos y factores estructurales presentes en la sociedad de recepción, que condicionan sus situaciones vitales. A través de este proceso culturalista, se generan acciones y discursos de control directo, desde las intervenciones sociales para disciplinar, encausar las lógicas de ser y estar de las mujeres inmigradas que encarnarían la diferencia social con la mayoría, con la norma.

En tercer lugar, en este llamado a la integración, aparece como un campo central, la incorporación al mercado laboral. En los discursos manifiestos en las investigaciones sobre la integración de las mujeres inmigradas se puede constatar que uno de los baremos centrales para considerar si un sujeto efectivamente está integrado, es el tipo de inserción laboral que manifiesta. Estar integrada al mercado laboral no es una posibilidad, sino una necesidad y obligación, emergiendo como una parte esencial de la norma social que se espera que las nuevas residentes acaten. Esto se visualiza pragmáticamente en que la mayoría de los servicios analizados, desarrolla algún tipo de acción directa para la inserción o la preparación sociolaboral.

De esta manera, el "trabajo" pasa a ser discursivamente una vía de inclusión efectiva y de movilidad social, lo que pone de relieve el énfasis liberal de los sistemas de intervención y la necesidad de sustentación del mercado económico, ya que su centro está en un cierto carácter utilitarista del fenómeno migratorio, donde se justifica su presencia mientras sea un aporte laboral al sistema. Gran parte de las definiciones de objetivos que desarrollan los servicios sociales analizados, se sitúan desde el inicio como espacios de preparación para una futura integración laboral, casi haciendo homólogos el significado de la integración con la inserción laboral de las mujeres, como vemos en el ejemplo:

En nuestros programas específicos de acogida también incorporamos la inserción laboral para mujeres inmigradas (...). Pero creemos que es central porque el trabajo es importante para insertarse. Tenemos este programa previo por si tienen alguna dificultad de inserción laboral relacionada con el hecho migratorio. Pueden ser temas del entorno, lingüísticos, por permisos de trabajo específicos o por permisos de residencia. O si tienen poco conocimiento del entorno. Las preparamos para poder después seguir un proceso de inserción laboral y que así tengan más posibilidades sociales efectivas. (Entrevista profesional. $\mathrm{ONG}$ ) 
Los dispositivos de intervención funcionan como mecanismos gubernamentales de gestión a través de los discursos y prácticas, en busca de que las mujeres migrantes se adapten a los sistemas lingüísticos, culturales y laborales de la sociedad de recepción, lo cual está asociado a su "correcta" integración.

\section{Integración en el entramado de relaciones de poder en la intervención}

Como se señaló, el ejercicio de control, disciplinamiento y normalización de este llamado a la integración implica asumir unas lógicas de diferenciación y de subalternización para poder hacerse efectiva.

El llamado a la integración que yace como telón de fondo en las intervenciones analizadas apela también a la voluntad de las mujeres en la gestión de sus cuerpos, costumbres, conocimientos y habilidades respecto de la sociedad en la que viven. De esta manera, las intervenciones públicas y de la sociedad civil desarrollan mecanismos de poder que partiendo desde la diferenciación, racializan y generizan a las mujeres inmigradas que asisten a esos servicios. Así, los sistemas de diferenciación basados en el género, la clase y la adscripción de etnia/origen nacional funcionan al unísono en la caracterización de la "mujer migrante" como un espacio sociosimbólico encarnado, donde se asentarán ejercicios reiterados de interpelación y disciplinamiento, en torno a los que se establecen ciertas relaciones de identidades, pertenencias y exclusiones (Romero Bachiller, 2007), y que permiten definir a estas personas como sujetos susceptibles de intervención. La configuración de la "mujer inmigrada", entonces, estará constituida a partir de las "carencias" que deberá superar a través de formación, ciertas disposiciones que deberá aprovechar para su inserción en determinados nichos laborales y de las costumbres que deberá modificar para su adaptación al contexto social.

Como señala Gregorio Gil (2012) la "construcción de la categoría «mujer(es) inmigrante(s)», no tendría por qué llevarnos a asignar pasividad a quienes están en esa posición de subalternidad y, mucho menos, colocarnos en una relación con ellas (ma)paternalista" (2012, p. 579). No obstante, desde los recursos sociales analizados, se propone implícitamente reparar y colmar esa falta con los distintos elementos que pueda proporcionar el servicio. La relación de intervención se vuelve así ejercicio de poder en el control y disciplinamiento de esos cuerpos y en la subjetivación que generan: en esta labor, los servicios emergen como instancias mediadoras entre la vida cotidiana de las mujeres inmigradas y la sociedad, a través los procesos de significación que llevan a cabo. Así, se produce una lógica que separa al servicio del exterior, situándolo como un ambiente de traducción entre las necesidades de las mujeres y las exigencias de la sociedad en términos de mercado de trabajo, conductas esperadas, participación social, etc. Esta noción de control y disciplina, la podemos visualizar en la cita siguiente:

Para mí el trabajo es preventivo y educativo, de acompañamiento, para que se inserten en la sociedad. Darles herramientas para que funcionen solas (...) un asesoramiento constante y después [de] que las mamás sepan atender como Dios manda a sus hijos, pequeñas cosas cotidianas, el día a día, que ellas sepan cómo funciona la educación aquí, todo el sistema, serían cosas muy importantes. (Entrevista profesional. ONG)

Vemos, en este ejemplo, una cierta lógica de dominación estructural y una supresión de la heterogeneidad de las mujeres inmigrantes (Mohanty, 2003). Siguiendo esta visión poscolonial, en algunos servicios se destaca una ubicación de la mujer blanca y europea en un estatus de superioridad respecto de las condiciones de género en comparación con las mujeres que asisten a los servicios. Ello se comprueba en los discursos de algunas interventoras que destacan la necesidad de liberar a estas mujeres inmigradas de las lógicas machistas que cargan, como si en el contexto local las desigualdades de género estuviesen ya superadas. Emerge una diferenciación jerarquizada, que tiene como uno de sus efectos la constitución de la categoría "mujer del tercer mundo" (Mohanty, 2003), un sujeto que -en la medida que es entendido como una "otra cultural"- es necesario educar en aras de 
su correcta incorporación a la sociedad de recepción, eludiendo las experiencias particulares y, en muchos casos, omitiendo las desigualdades sociales del contexto de instalación que afectan sus vidas. Por otra parte, también vemos que los esfuerzos de normalización y disciplina se vehiculan a través de roles de género que se articulan con la procedencia nacional para precisamente generar esa posibilidad de intervención en ámbitos privados y corporales. En relación con las disposiciones que se asumen como propias y auténticas de las mujeres inmigrantes, se entiende que estas personas son idóneas para tareas domésticas o de cuidado de personas, precisamente a partir del argumento referido a los roles tradicionales que ellas encarnan (Montenegro \& Montenegro, 2013). En ese sentido, considerando a Gregorio Gil, podemos señalar que "son las prácticas de poder, la colonialidad del poder, la que construye los cuerpos racializados, generizados y sexualizados, no la biología o el origen nacional" (Gregorio Gil, 2012, p. 585).

Como se señaló anteriormente, la diferencia cultural sirve como argumentación para este llamado a la integración, invisibilizando otros ejes de desigualdad que se articulan como pueden ser el género y la clase. El énfasis culturalista en las intervenciones sociales tiene efectos de etnización de las problemáticas sociales (Gregorio Gil \& Franze, 1999) que algunas mujeres inmigrantes llevan a los servicios analizados, pudiendo hacer que la inmigración sea vista exclusivamente a partir de esa diferencia cultural como un sector social diferenciado de otros.

Algunas de las actuaciones en los servicios hacen pensar que ciertos hábitos religiosos, preferencias culturales, hábitos familiares o formas de comunicación que tienen las mujeres atendidas son inadecuados, y se realizan acciones tendientes a su adecuación a lo esperado por el entorno social. Se intenta gobernarlas aplicando un disciplinamiento corporal en pos de una norma social esperable: así el cuerpo de las mujeres en los discursos de las interventoras aparece objetivizado, como manipulable y un contenido que debe ser modificado si se quiere acceder, por ejemplo, al mercado laboral. La diferencia, que debe ser erradicada, se conceptualiza como la relación a través de la que los grupos se posicionan en relación con el otro y no el reflejo objetivo del contenido de los grupos, sus valores y sus comportamientos (Bottero \& Irwin, 2003). Se induce, así, una forma de ser, pensar y actuar a las mujeres inmigradas de cómo ser "aceptable" al entorno social y laboral -sutil pero intenso-, como vemos en la siguiente cita:

Las mujeres musulmanas, al contrario de lo que pensamos, se arreglan mucho. Ellas quieren y van a su estilo y valoran mucho cuando les damos consejos de cómo situarse aquí en este contexto. En el día a día, les vamos explicando el uso de la ropa, a ellas les agrada que les demos esos consejos (...). Por ejemplo, con las jovencitas que llevan pañuelo negro, no les digo que no lo usen, pero les estoy diciendo que mejor negro no, y ahora lo llevan de colores. Si van a un trabajo, mejor blanco (...). Ellas aceptan las sugerencias. (Entrevista profesional. $\mathrm{ONG}$ )

De esta manera, se puede percibir cómo las intervenciones sociales analizadas generan efectos de control sobre los cuerpos de las mujeres migradas, en las formas de enunciarse y de visibilizarse, que en el cruce de género, clase y procedencia emergen visibles y se habla de ellos para acomodarlos y adaptarlos a las formas de hacer y de visualización de la mayoría. Con ello, el cuerpo de las mujeres inmigradas es visible simbólicamente en su condición de extranjeridad, mujer y en una posición económica desfavorable y en esa intersección de factores, su corporeidad es negada porque no es deseada para el conjunto social. El resultado del ejercicio de poder sobre dichas mujeres termina siendo la reproducción de condiciones para que el sistema las ubique en espacios de subordinación social. Porque el exhorto a la integración como una norma, no solo clasifica e intenta ordenar según las lógicas mayoritarias a las nuevas residentes del contexto español, sino que también las ubica en determinadas posiciones sociales, muchas veces, de subordinación y en espacios marginales. En el análisis vemos que, a través de los servicios, se reproduce la tendencia del mercado de trabajo generizado al promover la inserción laboral de las mujeres migradas casi exclusivamente en los ámbitos del cuidado (de personas dependientes, per- 
sonas mayores e infancia) y de servicios domésticos (Anthias \& Lazaradis, 2000; Bettio, Simonazzi, \& Villa, 2006) que muchas veces están precarizados. En estas formas de respuestas de los servicios, se invisibiliza la historia previa de las mujeres y sus niveles de formación, por lo tanto, en muchos casos, se produce un fenómeno de "desclasamiento social" de este sector. Así, la otredad cristalizada en la noción de mujer inmigrante pasa a ubicarse en las afueras del sistema y en una relación de subordinación económica y social, aunque su presencia implique la cohabitación (Galaz, 2009). Esto sucede, por ejemplo, en el hecho de que mujeres inmigradas con estudios universitarios o profesionales comienzan de cero en la formación para trabajos técnicos o de cuidados, sin considerar esa formación como base o capital social, como vemos en la cita:

Tenemos una población inmigrada muy bien preparada y no se está aprovechando. Una población inmigrada que tiene estudios (...) hay muchas mujeres que tienen estudios universitarios de su país. Pero tienen problemas de trámites que tienen que cruzar, las relaciones a nivel cotidiano son fuertes, no pueden acceder a otros trabajos que no sean los menos calificados. (Entrevista profesional. Servicio público)

Los entramados sociales, económicos y jurídicos en los que se insertan las intervenciones sociales con población inmigrada funcionan como exclusas en las que se priorizan ciertas trayectorias por las cuales las mujeres migrantes deberán circular y, en este contexto, las diversas formas de actuación de los servicios terminan preferentemente promoviendo una acomodación de las inmigradas en los bordes del sistema, en una posición de subalternización social. En ese sentido, las intervenciones están ajustadas a las lógicas contextuales, dentro de relaciones de poder neoliberales, en donde la urgencia por sustento social y económico les lleva a promover a esas mujeres en su ubicación en nichos laborales y educativos específicos y marginales.

Por otro lado, a partir del análisis en las investigaciones de los nichos laborales a los que pueden acceder, vemos que frecuentemente suplen los roles "feminizados" de las mujeres "autóctonas", posibilitando la "liberación de estas": se da un trasvase de las desigualdades de clase, género y procedencia (Juliano, 1998). De modo que las relaciones hegemónicas de género quedan incuestionadas como también las relaciones de poder económicas. Esto es, aunque las mujeres autóctonas quedan liberadas de las tareas de cuidado, las relaciones de poder por razones de género siguen activas. De modo que los valores de la sociedad patriarcal y de primacía económica se transmiten implícitamente en forma de norma (Bottero \& Irwin, 2003), reproduciendo la disposición social a partir de la diferencia de género que es difícil de cuestionar o modificar y, menos aún, cuando está inmersa en una asimetría de poder que es la relación entre "expertos/as" y "usuarios/as", y entre "nacionales" e "inmigrantes".

\section{Conclusiones}

A través de la argumentación planteada se evidencia de qué manera actúan los mecanismos de gubernamentalidad presentes en los servicios dirigidos a mujeres inmigradas, especialmente de qué forma se entrecruzan el control, la disciplina y la normalización, a través de la configuración de una “otredad problemática y carente”, lo que redunda en sistemas de inclusión social en los bordes del sistema. La configuración de la categoría "mujer inmigrante" está atravesada por criterios de género, culturales, geográficos y de clase que tienen como consecuencia la generación de un imaginario que legitima intervenciones que la ubican en una situación de subalternidad dentro del sistema y en condiciones de precariedad.

En los ejemplos de la sección donde se expuso cómo el exhorto a la integración se convierte en norma social, se muestra el énfasis de las intervenciones en tres áreas: lingüística, cultural y laboral. A partir de esta ejemplificación, se puede explicar cómo se constituye un entramado semiótico-material respecto del juego inclusión/exclusión, donde se espera que las personas inmigradas logren por sí mismas estar incluidas dentro del sistema a partir de unas herramientas técnicas, facilitadas desde las intervenciones. 
Esta inclusión se entiende como la adaptación de las personas migradas a las formas de hacer de la sociedad de recepción y, en especial, dentro de los espacios sociales, políticos, económicos y culturales que ésta define para los sujetos migrantes desde una posición hegemónica; por tanto, deben ser capaces de "habilitarse" en diversos frentes para poder insertarse de una manera "correcta" al nuevo medio.

Siguiendo a Rose (1996), esta forma de gobierno situaría la responsabilidad individual en la persona migrante que deberá gestionar sus saberes y sus comportamientos para integrarse adecuadamente al contexto de la sociedad de instalación. En el caso de las mujeres migrantes, está integración está marcada por factores de condición socioeconómica, etnia/origen nacional y género. En esta configuración, los servicios de atención actúan como espacios de regulación y disciplina de los cuerpos femeninos, en el sentido de que sus acciones y discursos se dirigen principalmente a "re-educar" a esas "otras culturales" para una supuesta correcta inclusión social. Esta re-educación implica una concepción disciplinaria (Foucault, 1975a) que permite conducir a distancia la conducta de los sujetos, a través de un encuadre social que se sistematiza en las acciones y discursos de las personas interventoras.

Así, en las intervenciones analizadas se pudo ver condensado un conjunto de prácticas y operaciones de gobierno para organizar el modus vivendi de las mujeres inmigradas, sin recurrir a coerciones directas (Foucault, 1975a), sino a través de lógicas de poder instauradas en la relación misma entre interventoras y usuarias. En los ejemplos expuestos del trabajo de campo, se buscó presentar nociones de cómo en ciertos discursos se reflejan algunos mecanismos de gubernamentalidad que moldean la conducta de las mujeres inmigradas; a través del control, la disciplina y la normalización de sus prácticas culturales y de sus cuerpos y la ubicación subalterna en espacios socioeconómicos. Todo ello, con prácticas sutiles dadas dentro de los espacios de contención -en los que se constituyen los servicios sociales para las mujeres inmigradas-, basándose en un conocimiento legitimado socialmente que se deriva de la posición de "expertas" de las interventoras. A través de ese conocimiento puesto en acción, podemos ver cómo los dispositivos de gobierno se cristalizan en las intervenciones sociales analizadas, teniendo el encargo de disciplinar a las usuarias respecto a lo que es esperado de ellas en la sociedad de instalación, a través de un proceso de categorización de clase, generizado y racializado (Deeb-Sossa, 2007; Mercer, Mohan, \& Power, 2003).

De esta manera, los servicios reproducen los imaginarios poscoloniales más amplios según los cuales los países emisores de inmigración están en condiciones de desarrollo menor respecto de la sociedad de recepción, una interpretación que explicaría por qué se asume que estas mujeres no tienen suficientes recursos personales para moverse en el entorno (Anthias, 2002; Suárez, 2004). Esto, a su vez, legitima la importancia de enfatizar en la formación y capacitación tanto práctica como valórica para su integración. Se solidifica de esta manera una noción de mujer inmigrada como un sujeto monolítico singular, una visión colonizada que supone, si seguimos a Mohanty (2003), una relación de dominación estructural y la supresión de la heterogeneidad de los/as sujetos, de sus voces, luchas y resistencias.

De esta manera, el lugar de las mujeres inmigradas se sitúa en la bisagra entre el adentro y el afuera, permitiendo la visibilidad y enunciación del grupo mayoritario como un "nosotras/os" situados/ as "dentro". El cuerpo de la mujer migrada aparece por el acento en la diferenciación de clase, racializada y generizada. Se sitúan afuera en su condición de extranjeridad no deseada, es decir de inmigrante, y en un adentro, pero en una inclusión "perversa" (Sawaia, 2002) o "diferencial", aquella que está sujeta "a variados grados de subordinación, reglas, discriminación y segmentación en un ámbito o esfera determinados" (Mezzadra \& Nielson, 2014, p. 14).

En el caso de las mujeres migrantes, se trata de una inclusión que prevé un lugar insostenible, incluida pero en los bordes del sistema donde gran parte no desea habitar: en espacios dejados por otras mujeres que han sido mal considerados y precarios. Ante la presión del mercado por obtener mano de obra barata, las exigencias gubernamentales y las propias demandas de urgencia social que levantan las mismas mujeres inmigradas, los servicios ter- 
minan por posibilitar una formación e inclusión en espacios subordinados del sistema, generando procesos de subalternización social (Dube, 2001). La efectividad de las relaciones de poder que se establecen dentro de los servicios se refuerza, como se dijo, a partir de la función compensatoria que desempeñan ante la negación y discriminación vivida por las usuarias en el contexto social, fuera de estos servicios sociales.

Es decir, que las mujeres inmigradas como grupo social, dada su posición en la estructura social, no están excluidas del sistema, sino que están incluidas en el mismo de manera perversa, esto es, a partir de la vulneración de sus derechos fundamentales. Porque como afirma Carmen Romero Bachiller:

Los efectos disciplinadores y normativizadores que se movilizan con el "reconocimiento" -que se construye sobre una cierta ficción-de las "mujeres inmigrantes" trabajan sistemáticamente en la incorporación de los "cuerpos-sujetos" así interpelados a los espacios delimitados como propios de "mujeres inmigrantes". Un espacio caracterizado por la vulnerabilidad, la discriminación y la denegación de los derechos vinculados a la posición de ciudadanía. (Romero Bachiller, 2007, p. 187)

\section{Referencias}

Agamben, G. (2011). ¿Qué es un dispositivo? Revista Sociológica, 26(73), 249-264.

Agrela, B. (2004). La acción social y las mujeres inmigrantes. ¿Hacia unos modelos de intervención? Portuaria, 4, 31-42.

Álvarez, S. (julio, 2008). Focopolítica y gubernamentalidad neoliberal, las políticas sociales. Trabajo presentado en el II Encuentro Argentino y Latinoamericano Prácticas Sociales y Pensamiento Crítico, Universidad Nacional de Córdoba. Recuperado de http://www.ets.unc.edu.ar/tercerencuentro/anteriores/2008/img/ datos/Universidad/alvarez.pdf

Anthias, F. (2002). Beyond feminism and multiculturalism: Locating difference and the politics of location. Women's Studies International Forum, 25(3), 275-286.
Anthias, F., \& Lazaradis, G. (Eds.). (2000). Gender and migration in southern Europe. Nueva York: Berg.

Batsleer, J., Chantler, K., \& Burman, E. (2003). Responses of health and social care staff to South Asian women who attempt suicide and/or self-harm. Journal of Social Work Practice, 17(1), 103-114.

Bettio, F., Simonazzi, A., \& Villa, P. (2006). Change in care regimes and female migration: The "care drain" in the Mediterranean. Journal of European Social Policy, 16(3), 271-285.

Blanch, A. (2004). La inmigración como problema: un análisis de las prácticas discursivas de la población autóctona. Papers, 74, 175-201.

Bottero, W., \& Irwin, S. (2003). Locating difference: Class, "race" and gender, and the shaping of social inequalities. The Sociological Review, 51(4), 463-483.

Brah, A. (1992). Diferencia, diversidad y diferenciación. En B. Hooks et al. (Eds.), Otras inapropiables. Feminismos desde las fronteras (pp. 107-136). Madrid: Traficantes de Sueños.

Callén, B., \& Montenegro, M. (2004). Inmigración y participación: hacia la construcción de espacios de convivencia vecinal. Encuentros en Psicología Social, 1(3), 217-223.

Carballeda, A. (2010). La intervención en lo social como dispositivo. Una mirada desde los escenarios actuales. Trabajo Social, 1, 46-59.

Deeb-Sossa, N. (2007). Helping the "neediest of the needy": An intersectional analysis of moral-identity construction at a community health clinic. Gender $\mathcal{E}$ Society, 21(5), 749-772.

Dube, S. (2001). Sujetos subalternos. México: El Colegio de México.

Foucault, M. (1975a). Vigilar y castigar. Nacimiento de la prisión. Madrid: Siglo XXI.

Foucault. M. (1975b). Defender la sociedad. Buenos Aires: FCE.

Foucault, M. (1977). El juego de Michel Foucault. En Saber y verdad (pp. 127-162). Madrid: Las Ediciones de la Piqueta.

Foucault, M. (1999). La gubernamentalidad. En Obras Esenciales: estética, ética y herméutica (Vol. 3, pp. 175-187). Barcelona: Paidós.

Galaz, C. (2009). Las relaciones de cooperación y exclusión entre personas con referentes socioculturales diversos. 
Un estudio sobre la alteridad (Tesis doctoral, Universidad Autónoma de Barcelona).

Gil, S. (2011). Nuevos feminismos. Sentidos comunes de la dispersión. Madrid: Traficantes de Sueños.

Goffman, E. (2006). La identidad deteriorada. Buenos Aires: Amorrortu.

Gregorio Gil, C. (1997). El estudio de las migraciones internacionales desde una perspectiva de género. Migraciones, 1, 145-175.

Gregorio Gil, C. (2010). Debates feministas en el análisis de la inmigración no comunitaria en el Estado español. Reflexiones desde la etnografía y la antropología social. Relaciones Internacionales, 14, 93-115.

Gregorio Gil, C. (2011). Fronteras de género y cultura en el contexto de la «fortaleza Europa». En D. Serrano-Niza (Coord.), iVisibles o invisibles? Mujeres migrantes, culturas y sociedades (pp. 55-67). Madrid: Plaza y Valdés.

Gregorio Gil, C. (2012). Tensiones conceptuales en la relación entre género y migraciones. Reflexiones desde la etnografía y la crítica feminista. Papers, 97(3), 569-590.

Gregorio Gil, C., \& Franze, A. (1999). Intervención social con población inmigrante: esos "otros"culturales". Intervención Psicosocial, 8(2), 163-175.

Grinberg, S. (2007). Gubernamentalidad: estudios y perspectivas. Revista Argentina de Sociología, 5(8), 95-110.

Hacking, I. (1999). The social construction of what? Cambridge, MA: Harvard University Press.

Haraway, D. (1991). Ciencia, cyborgs y mujeres. La reinvención de la naturaleza. Madrid: Ediciones Cátedra.

Herrera, E. (1994). Reflexiones en torno al concepto de integración en la sociología de la inmigración. Papers, 43, 71-76.

Ibáñez, T. (1994). Psicología social construccionista. Guadalajara: Universidad de Guadalajara.

Ibáñez, T. (1996). Fluctuaciones conceptuales en torno a la postmodernidad. Caracas: Universidad Central de Venezuela.

Juliano, D. (1994). Pobres mujeres o mujeres pobres. En C. Manzanos et al. (Eds.), Extranjeros en el paraíso (pp. 43-52). Barcelona: Virus.

Juliano, D. (1998). Las que saben. Subcultura de mujeres. Madrid: Horas y Horas.
Maquieira, V., Gregorio Gil, C., \& Gutiérrez Lima, E. (2000). Políticas públicas, género e inmigración. En P. Pérez Cantó (Ed.), También somos ciudadanas (pp. 371-442). Madrid: Instituto de Estudios de la Mujer.

Mezzadra, S., \& Nielson, B. (2014). Fronteras de inclusión diferencial. Subjetividad y luchas en el umbral de los excesos de justicia. Papeles del CEIC, 2(113), 1-30.Recuperado de: http://dx.doi.org/10.1387/ pceic. 12980

Mercer, C., Mohan, G., \& Power, M. (2003). Towards a critical political geography of African development. Geoforum, 34(4), 419-436.

Mohanty, C. (2003). Feminism without borders: Decolonizing theory, practicing solidarity. UK: DuKeU. Press.

Montenegro, M., \& Balasch, M. (2011). Conceptes i teories sobre problemes socials. En M. Montenegro (Coord.), Intervenció social. Controvèrsies teòriques i metodològiques (pp. 25-67). Barcelona: Editorial UOC.

Montenegro, M., Galaz, C., Yufra, L., \& Montenegro, K. (2011). Dinámicas de subjetivación y diferenciación en servicios sociales para mujeres inmigradas en la ciudad de Barcelona. Athenea Digital, 11(2), 113-132.

Montenegro, K., \& Montenegro, M. (2013). Governmentality in Service Provision for Migrated Women in Spain. Social and Personality Psychology Compass, 7(6), 331-342.

Nash, M. (2005). Doble alteridad en la comunidad imaginada de las mujeres inmigradas. En M. Nash, R. Tello \& N. Benach (Eds.), Inmigración, género y espacios urbanos. Los retos de la diversidad (pp. 1732). Barcelona: Edicions Bellaterra.

Parella, S. (2004). Mujer inmigrante y trabajadora: la triple discriminación. Barcelona: Anthropos.

Romero Bachiller, C. (2006). Articulaciones identitarias: prácticas y representaciones de género y "raza"| etnicidad en "mujeres inmigrantes" en el barrio de Embajadores (Madrid) (Tesis doctoral, Universidad Complutense de Madrid).

Romero Bachiller, C. (2007). El exotismo de los cuerpos y la fetichización de la mirada en la producción de las «mujeres inmigrantes» como «otras». En M. J. Sánchez Leyva \& A. Reigada (Coords.), Crítica 
feminista y comunicación (pp. 186-214). Sevilla: Comunicación Social.

Rose, N. (1996). Inventing ourselves: Psychology, power and personhood. Nueva York: Cambridge University Press.

Sandoval, J. (1997). Producción discursiva y problemas sociales. El ejemplo de la construcción social del problema del consumo de pasta base. Última Década, 7, 1-7.

Santamaría, E. (2002). La incógnita del extraño. Una aproximación a la significación sociológica de la «inmigración no comunitaria». Barcelona: Anthropos.

Sassen, S. (2013). Inmigrantes y ciudadanos. De las migraciones masivas a la Europa fortaleza. Madrid: Siglo XXI.

Sawaia, L. (2002). Uma jornada de humilhacoes: violência moral no trabalho. Brasil: Pontificia Universidad Católica de Sao Pablo.
Suárez, L. (2004). Rebordering the Mediterranean. Borders and citizenship in southern Europe. Oxford: Berghahn Books.

Subirats J., \& Alegre M. A. (Eds.). (2007). Educación e inmigración: nuevos retos para España en una perspectiva comparada. Madrid: Centro de Investigaciones Sociológicas.

Valles, M. S. (1999). Técnicas cualitativas de investigación

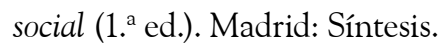

Wagman, D. (2006). Perfil racial en España: investigaciones y recomendaciones. Madrid: Grupo de Estudios y Alternativas 21.

Yufra, L. (2013). Inmigrantes en formación: estado de bienestar y promoción de lazos sociales en el caso de la formación para la inserción laboral de las personas inmigrantes en Barcelona. Athenea Digital, 13(2), 301-309. 
